\title{
A 500-750 GHz RF MEMS Waveguide Switch
}

\author{
Umer Shah, Member, IEEE, Theodore Reck, Senior Member, IEEE, Henrik Frid, Student Member, IEEE, \\ Cecile Jung-Kubiak, Member, IEEE, Goutam Chattopadhyay, Fellow, IEEE, Imran Mehdi, Fellow, IEEE, \\ and Joachim Oberhammer, Senior Member, IEEE
}

\begin{abstract}
This paper reports on a submillimeter-wave 500$750 \mathrm{GHz}$ MEMS waveguide switch based on a MEMSreconfigurable surface to block/unblock the wave propagation through the waveguide. In the non-blocking state the electromagnetic wave can pass freely through the MEMS-reconfigurable surface while in the blocking state the electric field lines of the TE $_{10}$ mode are short circuited which blocks the wave propagation through a WM-380 (WR-1.5) waveguide. A detailed design parameter study is carried out to determine the best combination of the number of horizontal bars and vertical columns of the MEMS-reconfigurable surface for achieving a low insertion loss in the non-blocking state and a high isolation in the blocking state for the 500-750 GHz band. Two different switch concepts relying on either an ohmic-contact or a capacitive-contact between the contact cantilevers have been implemented. The measurements of the switch prototypes show a superior RF performance of the capacitive-contact switch. The measured isolation of the capacitive-contact switch designed with $8 \mu \mathrm{m}$ contact overlap is 19 to $24 \mathrm{~dB}$ and the measured insertion loss in the non-blocking state is 2.5 to $3 \mathrm{~dB}$ from $500-750 \mathrm{GHz}$ including a $400 \mu \mathrm{m}$ long micromachined waveguide section. By measuring reference chips, it is shown that the MEMS-reconfigurable surface contributes only to 0.5 to $1 \mathrm{~dB}$ of the insertion loss while the rest is attributed to the limited sidewall metal thickness and to the surface roughness of the $400 \mu \mathrm{m}$ long micromachined waveguide section. Finally, reliability measurements in an uncontrolled laboratory environment on a comb-drive actuator with an actuation voltage of $28 \mathrm{~V}$ showed no degradation in the functioning of the actuator over one hundred million cycles. The actuator was also kept in the actuated state for $\mathbf{1 0}$ days and showed no sign of failure or deterioration.
\end{abstract}

Index Terms-RF MEMS, waveguide switch, micromachined waveguide, submillimeter-wave, rectangular waveguide, terahertz.

\section{INTRODUCTION}

W AVEGUIDES exhibit a very low insertion loss and a high power handling capability which makes them the preferred transmission media for high frequency and high power applications. Rectangular waveguides are the most commonly used waveguide type at millimetre- and submillimetrewave frequencies. These waveguides are usually fabricated from metals utilizing conventional metal machining techniques. However, for waveguides at WM-380 (WR-1.5) band and above, the typically specified fabrication accuracy is 15 $20 \mu \mathrm{m}$ [1], making the fabrication more difficult, costly and

Umer Shah, Henrik Frid and Joachim Oberhammer are with the Micro and Nanosystems, School of Electrical Engineering, KTH Royal Institute of Technology, Stockholm SE-100 44, Sweden (e-mail: umers@kth.se; joachimo@kth.se).

Theodore Reck, Cecile Jung-Kubiak, Goutam Chattopadhyay and Imran Mehdi are with the Jet Propulsion Laboratory, California Institute of Technology, Pasadena, CA 91109 USA (e-mail: Theodore.reck@jpl.nasa.gov). Manuscript received XXXXX; revised XXXXX. time consuming [2]. The dimensional accuracy requirements are even more severe for resonant structures [1]. As an alternative, micromachining can be employed to fabricate waveguides for submillimeter-wave frequency using silicon which is then metallized [3]. Silicon micromachined waveguides have already shown encouraging results, fabricated for applications at $2.7 \mathrm{THz}$ [4].

Microwave waveguide switches are used in waveguide based front-ends for signal routing, signal control, frequency band selection, beam scanning, calibration and redundancy applications. Conventional waveguide switch technology is mainly based on two concepts, namely, the mechanical switch concept using rotary motors and the semiconductor switch concept using p-i-n diodes and MESFETS integrated into the waveguide. The mechanical switches exhibit low insertion loss and high isolation but are bulky, heavy, require high power and have a very slow switching speed [5]. On the other hand, semiconductor switches are small in size and have a fast switching speed but exhibit poor insertion loss and isolation especially at higher frequencies [6]. Micorelectromechanical systems (MEMS) switches fabricated by micromachining have the potential to combine the advantages of both the mechanical and the semiconductor switches and replace the existing switch technology. This is due to the fact that RF MEMS switches offer very low insertion loss, high isolation, high linearity, large bandwidth of operation, low power consumption, switching speed in microsecond range and high miniaturization [7]. These switches have evolved and matured since their inception in the early 1990s and by now the major challenges they are facing are power handling and reliability [8]. At high input power levels, the power handling is limited by two mechanisms, namely the self-actuation in the open state and the latching in the closed state [9]. At submillimeter-wave frequencies, power handling is not an issue since at these frequencies only a few milliwatts power is typically available [10]. Reliability, on the other hand, is limited by dielectric charging for capacitive switches and by the contact physics for metal-contact micro-switches [11]. In recent publications [12], [13], layered contacts have been used to enhance reliability of metal contact switches by having a soft metal coated with a thin layer of a hard metal.

MEMS waveguide switches utilizing a ridge waveguide with electrothermal and electrostatic actuators have previously been reported for $\mathrm{Ku}$ and $\mathrm{K}$ band applications [14], [15]. More recently, an upper V-band waveguide switch design based on curled up MEMS actuators integrated inside the waveguide channel [16] has been shown, achieving a better than $20 \mathrm{~dB}$ isolation and good insertion loss performance. Furthermore, the authors have previously shown a very low 


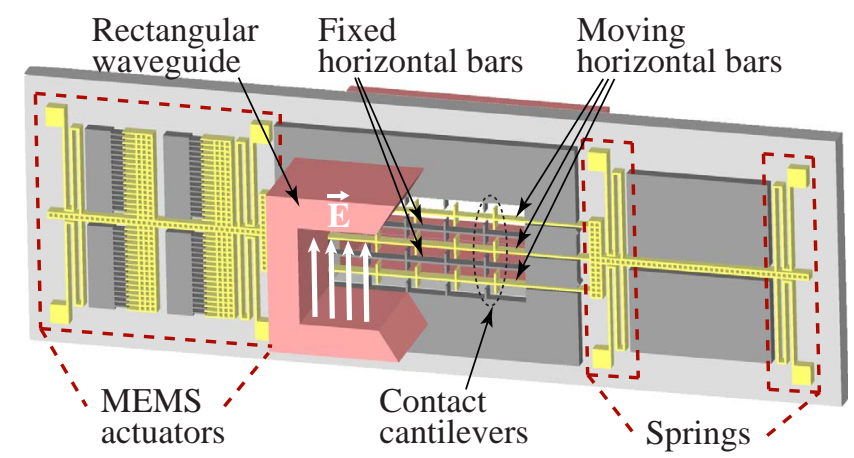

(a)

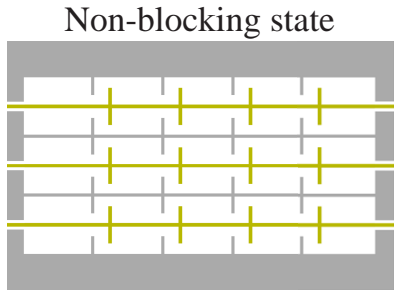

E-field free propagation

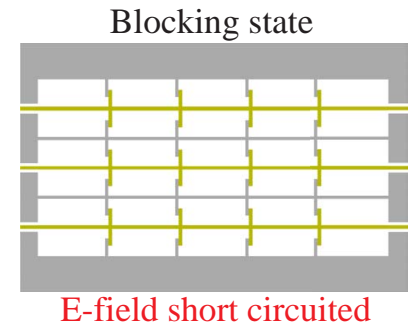

(b)

Fig. 1. Submillimeter-wave MEMS waveguide switch design using a MEMSreconfigurable surface: (a) 3-D illustration of the cross-section; and (b) nonblocking and blocking state of the MEMS waveguide switch.

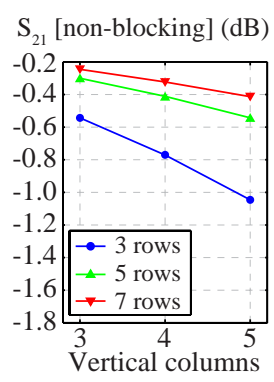

(a)

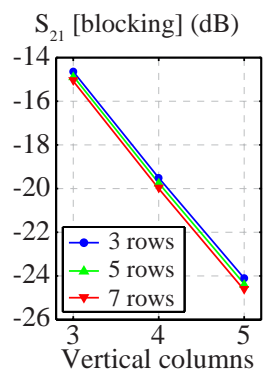

(b)

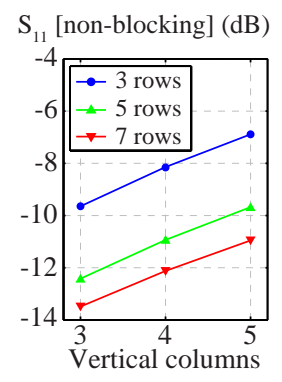

(c)
Fig. 2. Design parameter study at $625 \mathrm{GHz}$ for an ohmic-contact overlap of $2 \mu \mathrm{m}$ : (a) insertion loss in the non-blocking state; (b) isolation in the blocking state; and (c) return loss in the non-blocking state.

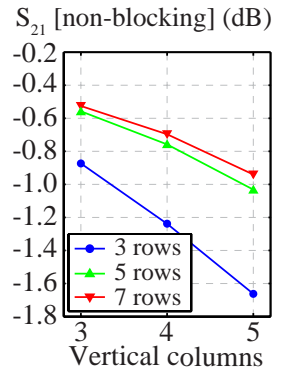

(a)

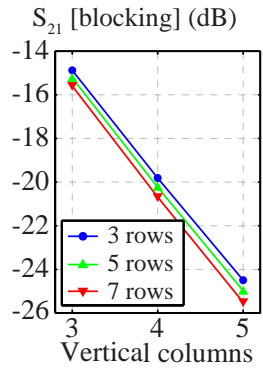

(b)

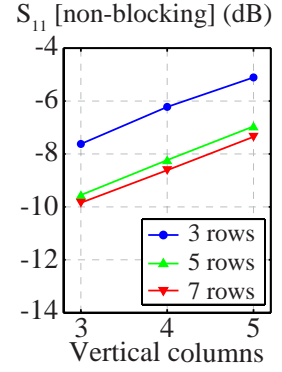

(c)
Fig. 3. Design parameter study at $625 \mathrm{GHz}$ for an ohmic-contact overlap of $8 \mu \mathrm{m}$ : (a) insertion loss in the non-blocking state; (b) isolation in the blocking state; and (c) return loss in the non-blocking state.

\section{CONCEPT AND DESIGN}

$30 \mathrm{~dB}$ ) MEMS waveguide switch for the upper V-band using a MEMS-reconfigurable surface to block the wave propagation in a rectangular waveguide [17], [18]. In a recent publication, the authors have used these MEMS-reconfigurable surfaces for switching waveguide stubs to implement a phase shifter operating in the frequency band between 500 to $550 \mathrm{GHz}$ [19] which was the highest frequency RF MEMS device ever reported so far.

The present paper introduces the first ever submillimeterwave MEMS waveguide switch operating in the frequency band between 500 to $750 \mathrm{GHz}$ using a MEMS-reconfigurable surface [17], [18] to block/unblock the wave propagation. This MEMS reconfigurable surface waveguide switch concept is very broadband, as the bandwidth is only limited by the waveguide-modes cutoff. The authors have reported, in a recent conference publication [20], the basic concept of this MEMS waveguide switch at WM-380 (WR-1.5) frequencies. This paper is an extension of the conference publication and discusses the switch design in detail and present new measurement data (both electromechanical and RF). To the best knowledge of the authors, the RF MEMS switch reported here is the first RF MEMS switch above $220 \mathrm{GHz}$ [21] and the first MEMS waveguide switch above $75 \mathrm{GHz}$. Very recently, another MEMS waveguide switch operating between 500 to $750 \mathrm{GHz}$ based on a ridge waveguide using an electrostatic actuator was reported [22] and our conference publication [20] was referenced as the first MEMS waveguide switch at these frequencies by that paper.
The waveguide switch reported in this paper comprises a single-pole single-throw (SPST) design utilizing a MEMSreconfigurable surface inserted in the cross-sectional plane of the rectangular waveguide as shown in Fig. 1. The use of the MEMS-reconfigurable surface for blocking/unblocking the wave propagation into a waveguide has already been shown by the authors [17], [18] for V-band. Fig. 1(b) shows the two states of the MEMS waveguide switch. In the non-blocking state, the gap between the contact cantilevers allows for the reconfigurable surface and in the blocking state, the movable contact cantilevers are moved to contact the fixed contact cantilevers to form a series of vertical columns which short circuit the electric field lines of the $\mathrm{TE}_{10}$ mode blocking the electromagnetic wave propagation.

The overall switch performance is influenced by the number of horizontal bars (rows), the number of vertical contact cantilevers (columns) and the overlap of the contact cantilevers. The performance variation based on these model parameters are simulated using full-wave simulations in CST Microwave Studio and are shown in Fig. 2 and Fig. 3 to find the best combination of the model parameters for the switch design for 500-750 GHz, i.e., ten times higher frequency than previous designs. The simulation results show that the isolation in the blocking state improves while the insertion loss in the nonblocking state degrades with increasing number of vertical columns [Fig. 2, Fig. 3]. The number of horizontal bars electromagnetic wave to propagate freely through the MEMS- 
has a minimal impact on the isolation performance in the blocking state since the horizontal bars are perpendicular to the short circuited electric field lines. In the non-blocking state of the switch, the insertion loss is significantly higher when three horizontal bars are used instead of four or five horizontal bars. This is an indirect effect resulting from the larger contact cantilever length used for a switch design with lower number of horizontal bars to maintain the same contact overlap. The longer contact cantilevers adversely affect the waveguide electric field lines in the non-blocking sate since the contact cantilevers are parallel to the field lines. The degradation of the insertion loss with increasing length of the contact cantilevers is also observed when the contact overlap is increased from $2 \mu \mathrm{m}$ to $8 \mu \mathrm{m}$ as shown in Fig. 2(a) and Fig. 3(a), respectively. This design parameter study makes it clear that for the optimal switch design, the selection of the number of horizontal bars and vertical columns requires a compromise between insertion loss, isolation and the return loss. Based on the simulation results, the design with 7 horizontal bars and 4 vertical columns is selected for a $2 \mu \mathrm{m}$ contact overlap since it has the non-blocking state insertion and return loss below $0.4 \mathrm{~dB}$ and $12 \mathrm{~dB}$, respectively, and the blocking state isolation above $20 \mathrm{~dB}$.

It has been shown by the authors in a recent publication [19] that due to fabrication inaccuracies, an ohmic contact cannot be guaranteed for all individual switch contacts simultaneously. For the design with $2 \mu \mathrm{m}$ contact overlap, each contact cantilever has a length of $11 \mu \mathrm{m}$ with a $4 \mu \mathrm{m}$ width resulting in a spring constant of $60900 \mathrm{~N} / \mathrm{m}$ which limits the possible movement of all further cantilevers into contact to only $0.5 \mathrm{~nm}$ after the first contact is closed. Thus, with the implemented actuator design, closing of all further switch contacts is not guaranteed. In contrast, the spring constant of the contact cantilevers for the V-band ohmic-contact MEMS waveguide switch design published previously by the authors was only $170 \mathrm{~N} / \mathrm{m}$ [17], [18] which is much more compliant and allows for a possible further movement of $200 \mathrm{~nm}$ after the first contact is closed. To overcome this issue the contact overlap was increased from 2 to $8 \mu \mathrm{m}$. This results in the required isolation being achieved in the blocking state even for a small air gap between the contact cantilevers i.e., a capacitive contact is sufficient and a metal contact is not required. This increased overlap adversely influences the non-blocking state return loss as shown in Fig. 2(c) and Fig. 3(c), which is a design compromise. Therefore, a second switch design, herein referred to as capacitive-contact waveguide switch in contrast to the $2 \mu \mathrm{m}$ overlap ohmic-contact switch, with $8 \mu \mathrm{m}$ contact overlap using 5 horizontal bars and 4 vertical columns achieving a simulated insertion loss below $0.8 \mathrm{~dB}$, isolation above $20 \mathrm{~dB}$ and the return loss below $8 \mathrm{~dB}$ was also fabricated.

Moreover, the authors have shown in the previous V-band waveguide switch publication [18] that a strong sinusoidal configuration of the contact cantilevers in the waveguide crosssection achieves a similar overall performance for a reduced number of contact points. For the current WM-380 (WR1.5) waveguide switch design, the waveguide dimensions are such that the design with the maximum number of contact cantilevers only has a total of 24 contact points instead of

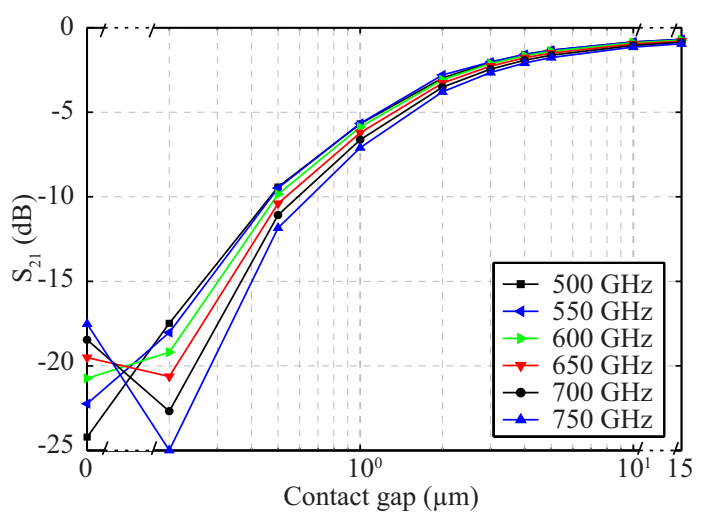

Fig. 4. Simulation results of the design parameter study to evaluate the switch isolation when having a capacitive contact instead of an ohmic contact for a contact overlap of $8 \mu \mathrm{m}$ using 5 horizontal bars and 4 vertical columns.

440 contact points used in [18]. Any further reduction in this number of contact points is not possible while simultaneously maintaining similar performance of the blocking state isolation and non-blocking state insertion and return loss.

Fig. 4 shows $S_{21}$ as a function of the contact gap for a switch design with $8 \mu \mathrm{m}$ contact overlap using 5 horizontal bars and 4 vertical columns. The desired isolation is achieved even for a contact gap of $200 \mathrm{~nm}$ when the overlap between the contact cantilevers is $8 \mu \mathrm{m}$. This gap is used in further simulations in this paper to better model the contact between the contact cantilevers in the blocking state. Furthermore, a $15 \mu \mathrm{m}$ initial contact gap was chosen for the non-blocking state.

The MEMS switch has standard WM-380 (WR-1.5) rectangular waveguide dimensions $(0.380 \mathrm{~mm} \times 0.190 \mathrm{~mm})$ with the electrostatic comb-drive MEMS actuator having 160 fingers placed on one side of the narrow wall of the waveguide using four folded-beam springs as suspension beams each with a length of $700 \mu \mathrm{m}$ symmetrically placed around the narrow wall of the waveguide opening. Four different comb-drive designs with different spring widths and finger gaps achieving spring constant variations of $1.06,1.69$ and $3.59 \mathrm{~N} / \mathrm{m}$ were implemented as actuator variations.

\section{FABRICATION AND AsSEMbly}

The assembled switch consists of a MEMS waveguide switch chip with the MEMS-reconfigurable surface and a top silicon chip which is used to align the MEMS waveguide switch chip to the waveguide flanges. The MEMS waveguide switch chips are fabricated using a silicon-on-insulator (SOI) wafer in a two mask micromachining process developed by the authors [17], [18]. The fabricated chips are metallized by sputtering a gold layer thickness of $700 \mathrm{~nm}$ on the SOI handle wafer and a gold layer thickness of $200 \mathrm{~nm}$ on the SOI device layer. Fig. 5 illustrates a close-up SEM image of a single MEMS waveguide switch chip showing the MEMS-reconfigurable surface, the comb-drive actuators and the mechanical springs. The total dimensions of the MEMS waveguide switch elements are $1.6 \mathrm{~mm} \times 1.5 \mathrm{~mm}$. The processed wafer had a total of 112 MEMS waveguide switch 


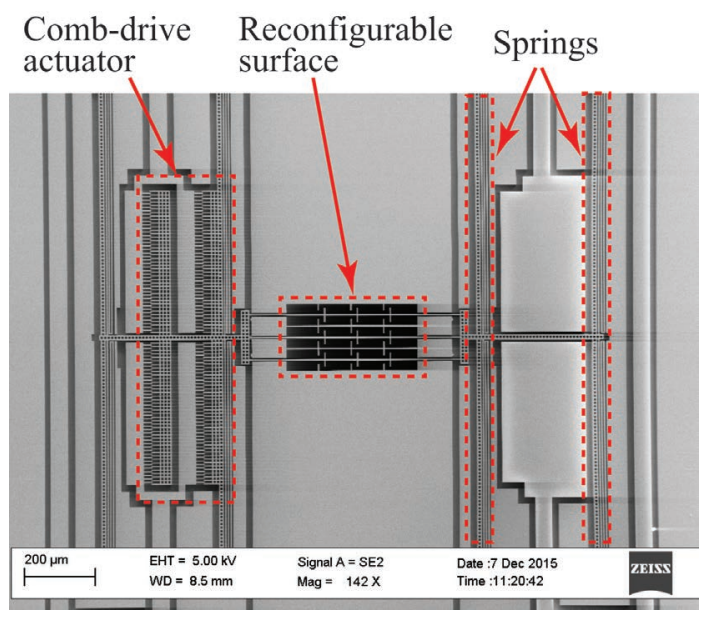

Fig. 5. Close-up SEM image of the fabricated MEMS waveguide switch chip with MEMS-reconfigurable surface and the comb-drive actuators.

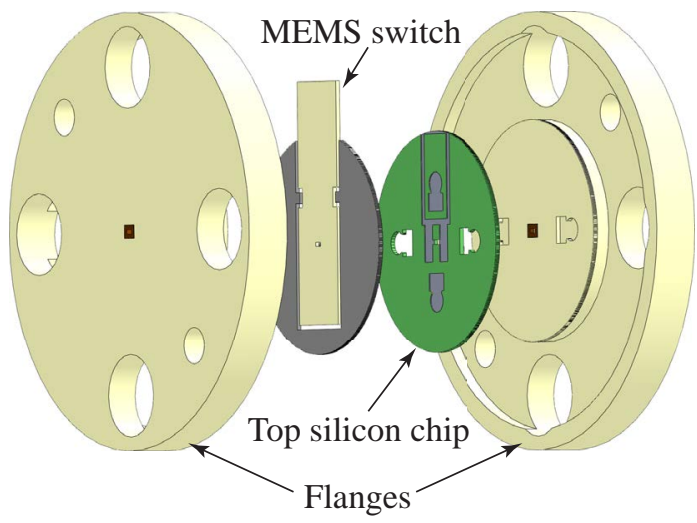

(a)

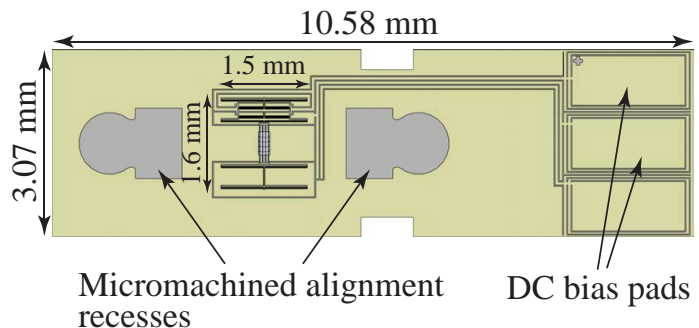

(b)

Fig. 6. MEMS waveguide switch: (a) exploded-view drawing showing the mounting/assembly of the MEMS waveguide switch into the WR-1.5 waveguide flanges; and (b) illustration of the complete MEMS waveguide switch chip.

chips achieving a process yield of between $90-95 \%$ during fabrication in a university cleanroom.

Fig. 6(a) shows the exploded-view drawing illustrating the mounting/assembly procedure of the MEMS waveguide switch and Fig. 6(b) shows a drawing of the entire MEMS waveguide switch chip of overall dimensions of $3.07 \mathrm{~mm} \times 10.58 \mathrm{~mm}$, including the DC bias lines, bias pads and alignment recesses. The waveguide flanges, the MEMS waveguide switch chip and the top silicon chip have omega-shaped alignment recesses where omega-shaped springs are inserted for accurate alignment between the metal and the silicon pieces [23].

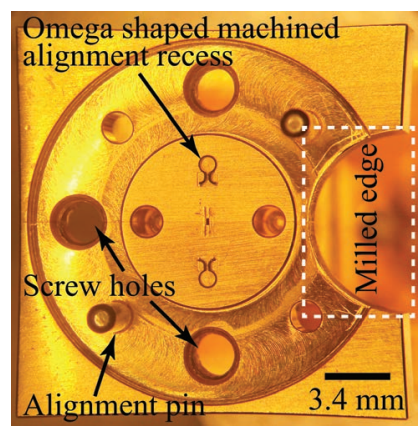

(a)

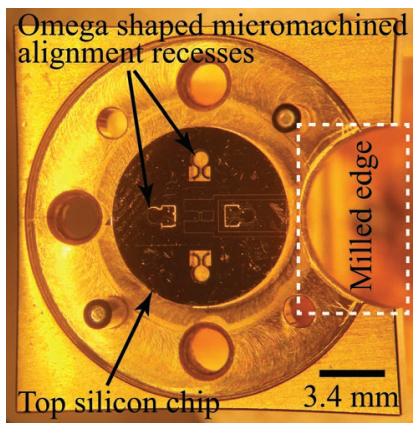

(b)

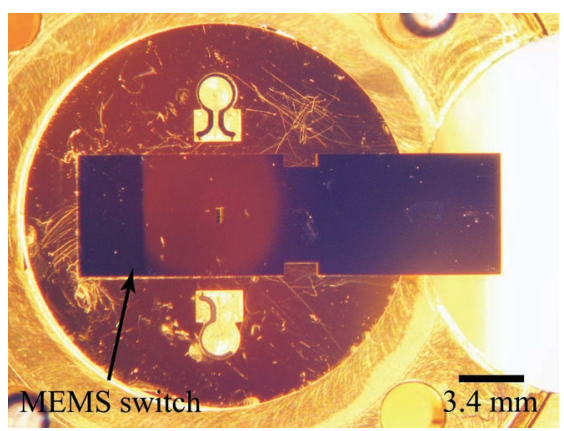

(c)

Fig. 7. Assembly/mounting of the MEMS waveguide switch: (a) waveguide flange with omega springs inserted into the omega shaped micromachined alignment recesses; (b) placement of the top silicon chip; and (c) placement of the MEMS waveguide switch chip.

Fig. 7 show microscope pictures of the mounting/assembling of the MEMS waveguide switch. Firstly, omega-shaped silicon springs are placed in the machined alignment recesses on the waveguide flange [Fig. 7(a)]. This is followed by alignment and placement of the top silicon chip on the waveguide flange [Fig. 7(b)]. Next, omega-shaped silicon springs are placed in the etched micromachined alignment recesses on the top silicon chip and the MEMS waveguide switch chip is aligned and placed on top of the top silicon chip [Fig. 7(c)]. Finally, the second waveguide flange is placed on top and the whole assembly is screwed together. One of the waveguide flanges has an edge removed on one side [Fig. 7], so that the DC bias pads on the MEMS waveguide switch chip can be accessed by DC probes.

\section{Characterization AND AnALysis}

\section{A. Submillimeter-wave Characterization}

The submillimeter-wave measurements of the MEMS waveguide switch prototypes were performed with an Agilent PNAX system using VDI WR1.5 (WM-380)-VNAX Extenders calibrated using SOLT calibration. Two different MEMS waveguide switch concepts have been implemented: (1) capacitive-contact switch with a contact overlap of $8 \mu \mathrm{m}$ for which capacitive, i.e., proximity, contact between the cantilevers is sufficient; and (2) an ohmic-contact switch with a contact overlap of $2 \mu \mathrm{m}$ relying on metal contact between the cantilevers, i.e., physical touching of all contact points and sufficiently low contact resistance. 


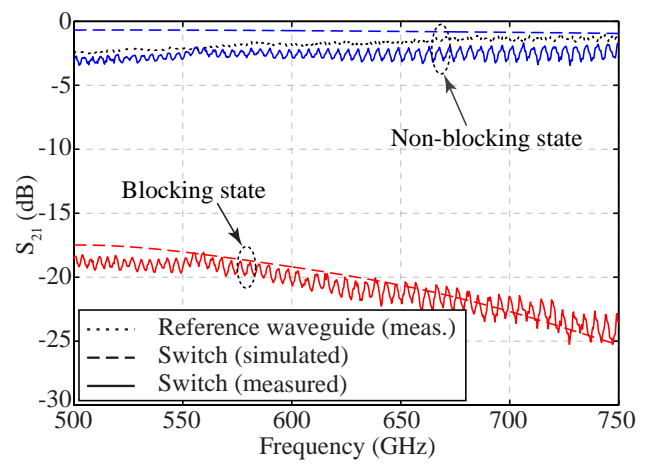

(a)

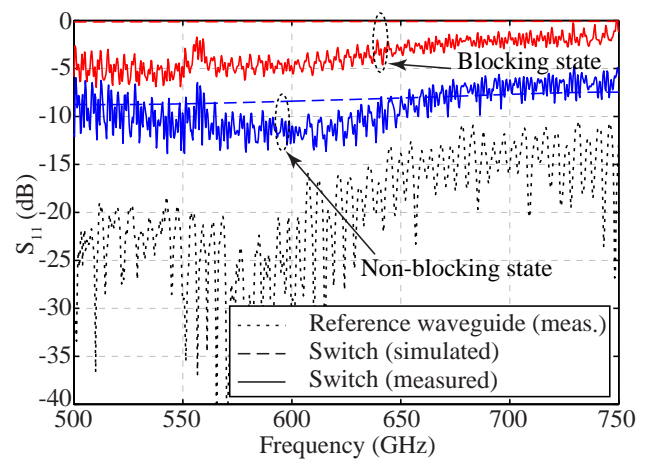

(b)

Fig. 8. Measured and simulated S-parameters of the capacitive-contact MEMS waveguide switch with contact overlap of $8 \mu \mathrm{m}$ having 5 horizontal bars and 4 vertical columns: (a) $\mathrm{S}_{21}$; and (b) $\mathrm{S}_{11}$. The reference waveguide measurement shows the measurement of micromachined hollow straight reference waveguide without any MEMS surfaces.

1) Capacitive-Contact Switch: Fig. 8 shows the simulated and measured insertion and return loss for the capacitivecontact MEMS waveguide switch design with a $8 \mu \mathrm{m}$ contact overlap having 5 horizontal bars and 4 vertical columns. The calibration is done between the two waveguide flanges without any MEMS chip. The broadband frequency measurements between $500-750 \mathrm{GHz}$ show that the blocking state isolation $\left(\mathrm{S}_{21}\right)$ is better than $19 \mathrm{~dB}$ and even reaching $24 \mathrm{~dB}$ at $750 \mathrm{GHz}$. This is well matched with the simulation results from CST Microwave Studio where a $200 \mathrm{~nm}$ gap between the contacts is used for the blocking state with a simulated insertion loss of $17.5 \mathrm{~dB}(500 \mathrm{GHz})$ to $25 \mathrm{~dB}(750 \mathrm{GHz})$. The measured insertion loss in the non-blocking state is around $2.2 \mathrm{~dB}$ higher when compared to the simulations which is assumed to be partially attributed to the small gaps in the chip assembly which result in a significant increase in the insertion loss already shown by the authors in [19]. In addition, the higher insertion loss is also due to limited sidewall gold coverage during sputtering and higher surface roughness during deep reactive ion etching (DRIE) of the $400 \mu \mathrm{m}$ long micromachined waveguide section. This waveguide section is formed by etching the handle layer (thickness $=400 \mu \mathrm{m}$ ) of the SOI wafer used for fabrication. Using an SOI wafer with a thinner handle layer could improve the insertion loss as shown for the ohmic-contact MEMS waveguide switch where a $300 \mu \mathrm{m}$ SOI handle wafer was used instead. Fig. 9 shows the cross-sectional SEM image of the MEMS waveguide switch chip. The close-

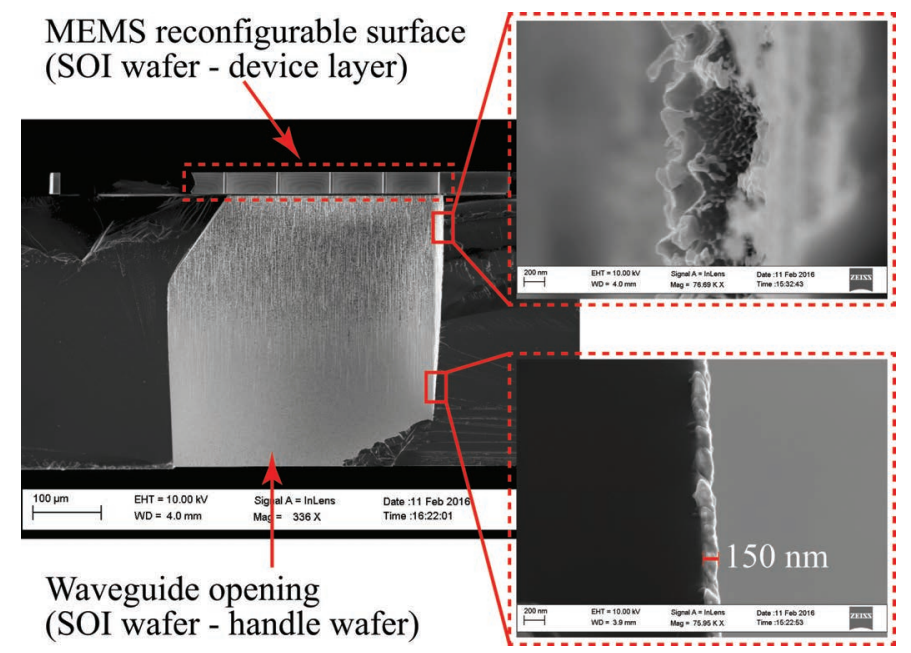

Fig. 9. Cross-sectional SEM image of the fabricated capacitive-contact MEMS waveguide switch with contact overlap of $8 \mu \mathrm{m}$. Close-up of the top and bottom waveguide sidewalls are shown.

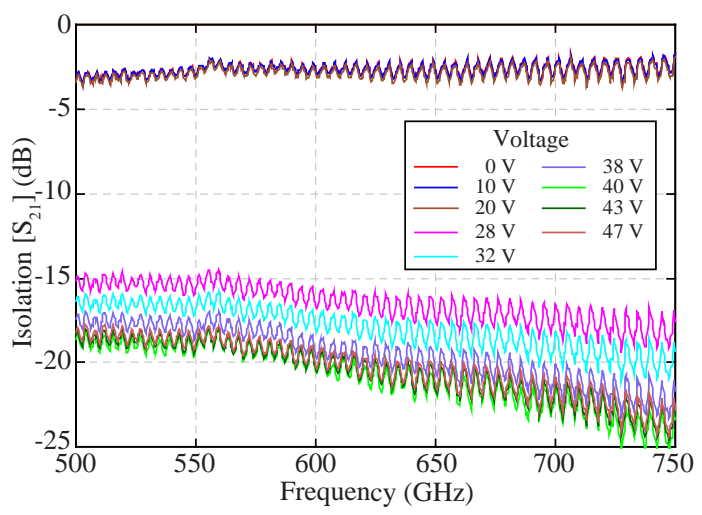

Fig. 10. Measured isolation as a function of actuation voltage for the capacitive-contact MEMS waveguide switch with contact overlap of $8 \mu \mathrm{m}$ having 5 horizontal bars and 4 vertical columns.

up inserts illustrate a uniform metal coverage with a thickness of $150 \mathrm{~nm}$ at the top of the micromachined waveguide section and non-uniform metal coverage at the bottom. The higher surface roughness is also clearly visible at larger etch depths of the waveguide section. The contribution to the insertion loss by the MEMS-reconfigurable surface is only about 0.5 to 1 $\mathrm{dB}$ which is verified by the reference waveguide measurement of a straight micromachined waveguide without the MEMSreconfigurable surface. The loss factor $\left(1-\left|S_{11}\right|^{2}-\left|S_{21}\right|^{2}\right)$ for this switch in the blocking state is 0.65 at $500 \mathrm{GHz}$ and going down to 0.3 at $750 \mathrm{GHz}$. For the non-blocking state, the values are 0.4 at $500 \mathrm{GHz}$ and 0.2 at $750 \mathrm{GHz}$.

Fig. 10 shows the measured isolation $\left(\mathrm{S}_{21}\right)$ as a function of actuation voltage for the capacitive-contact MEMS waveguide switch design with a $8 \mu \mathrm{m}$ contact overlap having 5 horizontal bars and 4 vertical columns. There is a minimal change in the isolation $\left(\mathrm{S}_{21}\right)$ when the actuation voltage is increased from $0 \mathrm{~V}$ to $20 \mathrm{~V}$. From $20 \mathrm{~V}$ to $28 \mathrm{~V}$, the isolation $\left(\mathrm{S}_{21}\right)$ increases to above $15 \mathrm{~dB}$. At $40 \mathrm{~V}$, the first contact points are touching and any further increase of the actuation voltage does not increase the isolation $\left(\mathrm{S}_{21}\right)$. 


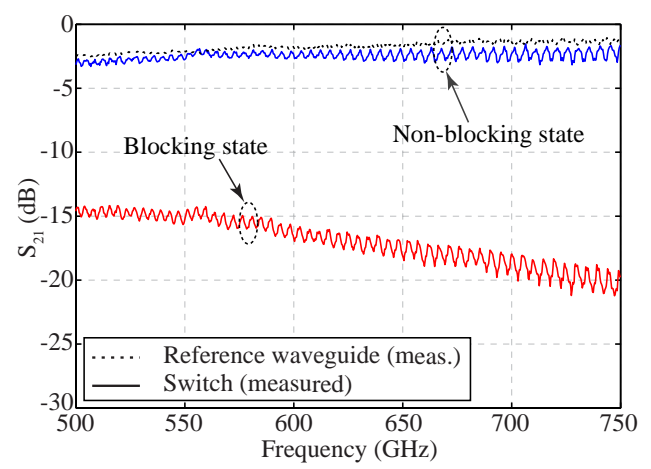

(a)

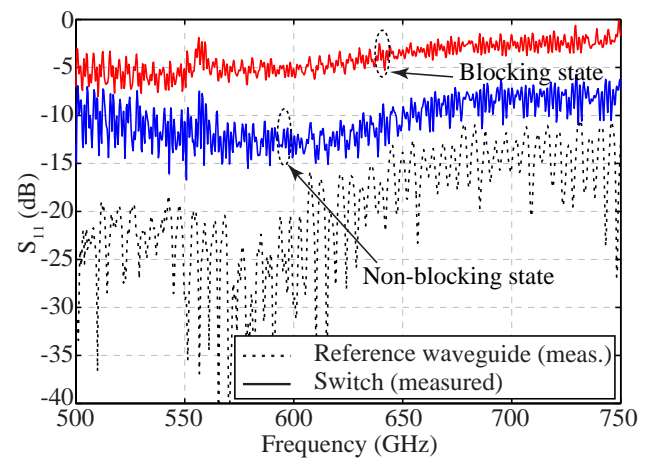

(b)

Fig. 11. Measured S-parameters of the capacitive-contact MEMS waveguide switch with contact overlap of $8 \mu \mathrm{m}$ having 5 horizontal bars and 3 vertical columns: (a) $\mathrm{S}_{21}$; and (b) $\mathrm{S}_{11}$. The reference waveguide measurement shows the measurement of micromachined hollow straight reference waveguide without any MEMS surfaces.

Fig. 11 shows the measured insertion and return loss for a design variation of the capacitive-contact MEMS waveguide switch having 5 horizontal bars and 3 vertical columns. The isolation in the blocking state is reduced by around $4 \mathrm{~dB}$ when the number of vertical columns is reduced from 4 [Fig. 8] to 3 [Fig. 11]. This confirms the CST simulations shown in Fig. 3. The improvement in the insertion and return loss in the non-blocking state as predicted by CST simulations in Fig. 3 is not observed in the measurements which is due to the overall losses being dominated by the loss of the $400 \mu \mathrm{m}$ long micromachined waveguide section. The measured loss factor for this switch in the blocking state is 0.65 at $500 \mathrm{GHz}$ and going down to 0.3 at $750 \mathrm{GHz}$. For the non-blocking state, the values are 0.4 at $500 \mathrm{GHz}$ and 0.2 at $750 \mathrm{GHz}$.

2) Ohmic-Contact Switch: Fig. 12 shows the simulated and measured insertion and return loss for the ohmic-contact MEMS waveguide switch design with a $2 \mu \mathrm{m}$ contact cantilever overlap having 7 horizontal bars and 4 vertical columns. The switch has a broadband frequency response with the insertion loss of around $1.2 \mathrm{~dB}$ in the non-blocking state for the design frequency range of 500-750 GHz. The measured insertion loss in the non-blocking state is $1.3 \mathrm{~dB}$ better than for the capacitive-contact switch [Fig. 8] which is due to the combination of using a shorter contact cantilever length [Fig. 2(a), Fig. 3(a)] and the fact that a thinner SOI wafer with handle layer thickness of $300 \mu \mathrm{m}$ was used in the fabrication. However, the measured switch isolation $\left(S_{21}\right)$ in the blocking

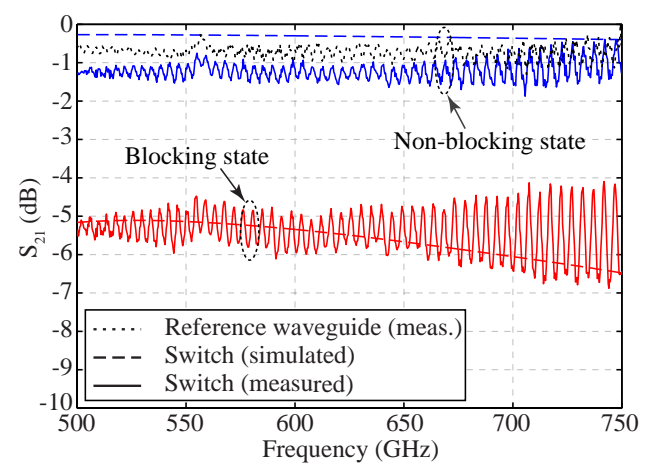

(a)

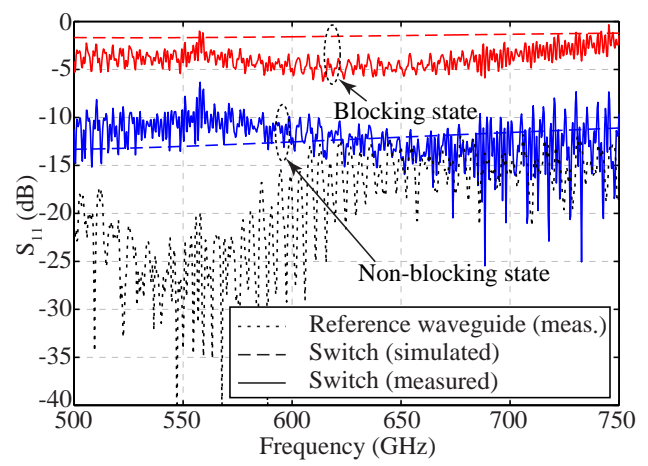

(b)

Fig. 12. Measured and simulated S-parameters of the ohmic-contact MEMS waveguide switch with contact overlap of $2 \mu \mathrm{m}$ having 7 horizontal bars and 4 vertical columns: (a) $\mathrm{S}_{21}$; and (b) $\mathrm{S}_{11}$.

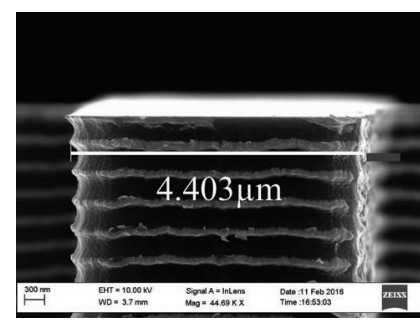

(a)

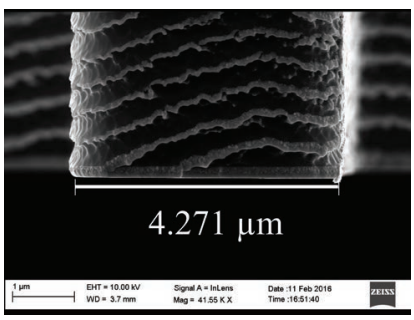

(b)
Fig. 13. Cross-sectional SEM image of the contact cantilevers, showing a sidewall inclination of $0.25^{\circ}$ : (a) top part of the cantilever; and (b) bottom part of the cantilever.

state is only about $5 \mathrm{~dB}$. This is due to the fabrication irregularities resulting in decreased probability of all contacts closing which is required for proper operation of the metal-contact switch. Fig. 13 shows fabrication inaccuracies by illustrating the cross-sectional SEM images of the top and bottom part of the contact cantilevers showing a sidewall scallop size of $150-200 \mathrm{~nm}$ in the deep reactive ion etched device layer. In addition, the contact cantilever sidewall profile is not straight and a sidewall angle of $0.25^{\circ}$ is measured from the crosssectional SEM images. To take these fabrication inaccuracies into account in the model, a $200 \mathrm{~nm}$ gap between the contact cantilevers was therefore used in simulations for the blocking state. The measured loss factor for this switch in the blocking state is 0.3 at $500 \mathrm{GHz}$ and going down to 0.1 at $750 \mathrm{GHz}$. For the non-blocking state, the values are 0.2 at $500 \mathrm{GHz}$ and 0.1 at $750 \mathrm{GHz}$. 
TABLE I

ACTUATOR DESIGN AND CHARACTERIZATION

\begin{tabular}{|lcccc|}
\hline $\begin{array}{l}\text { Actuator } \\
\text { No. }\end{array}$ & $\begin{array}{c}\text { Spring } \\
\text { Width } \\
{[\mu \mathrm{m}]}\end{array}$ & $\begin{array}{c}\text { Gap } \\
\text { Between } \\
\text { Fingers } \\
{[\mu \mathrm{m}]}\end{array}$ & $\begin{array}{c}\text { Spring } \\
\text { Constant } \\
{[\mathrm{N} / \mathrm{m}]}\end{array}$ & $\begin{array}{c}\text { Measured } \\
\text { Actuation } \\
\text { Voltage } \\
{[\mathrm{V}]}\end{array}$ \\
\hline \hline 1 & 3 & 2 & 1.06 & 28 \\
2 & 3 & 2.5 & 1.06 & 36 \\
3 & 3.5 & 2.5 & 1.69 & 40 \\
4 & 4.5 & 2 & 3.59 & 49 \\
\hline
\end{tabular}

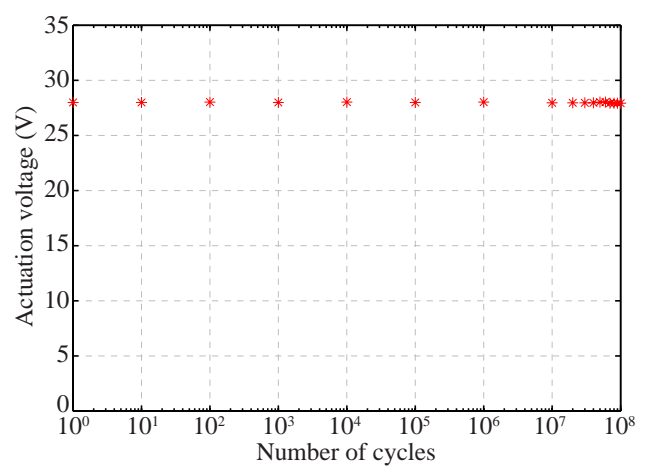

Fig. 14. Life-time measurements with the actuation voltage being monitored over 100 million cycles using a $36 \mathrm{~V}$ unipolar square waveform with a $50 \%$ duty cycle and a cycle frequency of $100 \mathrm{~Hz}$.

\section{B. Electromechanical Characterization and Reliability}

The design parameters used for the four implemented comb drive actuators are summarized in Table I together with the measured actuation voltages. Actuator 1 was chosen for further detailed electromechanical characterization. The actuation voltage repeatability measurement shows that for 20 consecutive actuation cycles, the average actuation voltage is $27.99 \mathrm{~V}$ with a standard deviation of $44.7 \mathrm{mV}$.

For performing the lifetime measurements, actuator 1 [Table I] was cycled using a unipolar square waveform with a $50 \%$ duty cycle at a frequency of $100 \mathrm{~Hz}$ with an actuation voltage of $36 \mathrm{~V}$ ( $28 \%$ over-voltage drive). Fig. 14 shows the result of the lifetime measurements where the actuation voltage was measured after each decade of actuation cycles and remains stable at $28 \mathrm{~V}$ with no significant variation over the measured one hundred million cycles (standard deviation of all 45 measurement points is $66.1 \mathrm{mV}$ ), performed even in an uncontrolled laboratory environment.

As a further reliability test of the switch design, long term actuation measurements were performed on actuator 1 . The actuator was held in a continuous actuated state for ten days with a voltage of $36 \mathrm{~V}$ and only once per day the actuation voltage was measured by averaging the results from three consecutive actuation-voltage ramping cycles at each measurement point. Fig. 15 summarizes the results and shows a very low variation in the measured average actuation voltage over the ten day forced-ON period. The actuator remained fully operational during and after the measurement tests without any sign of failure or degradation.

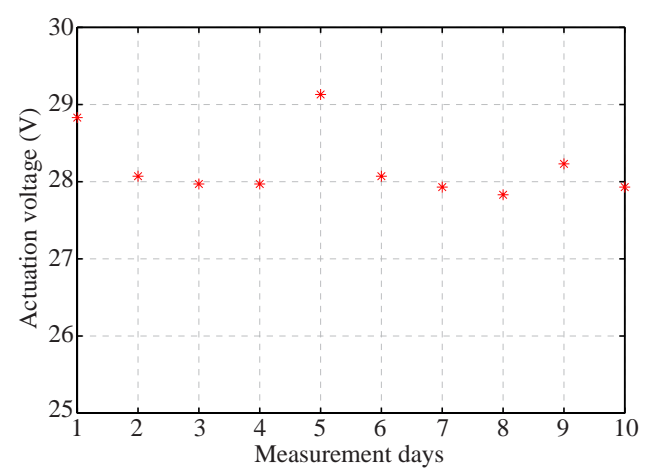

Fig. 15. Long term continuous actuated state measurements using a voltage of $36 \mathrm{~V}$, with the actuation voltage monitored once per day.

\section{CONClusion}

We have reported the design, fabrication and detailed characterization of the first submillimeter-wave $500-750 \mathrm{GHz}$ MEMS waveguide switch comprising a MEMS-reconfigurable surface for blocking/unblocking the wave propagation through a waveguide. Detailed design parameter analysis was performed to select the best design parameters for achieving a low insertion loss in the non-blocking state and a high isolation in the blocking state. Two different switch concepts based on an ohmic-contact and a capacitive-contact between the contact cantilevers were compared. The ohmic-contact waveguide switch did not work properly in the blocking state achieving and isolation of only $5 \mathrm{~dB}$. On the other hand, the capacitivecontact waveguide switch achieved an isolation of 19-24 dB and an insertion loss of 2.5-3 dB which included losses from a $400 \mu \mathrm{m}$ long micromachined waveguide section. The major part of the insertion loss is attributed to insufficient gold coverage and surface roughness of the waveguide sidewalls and only 0.5 to $1 \mathrm{~dB}$ is attributed to the MEMS-reconfigurable surface. In addition, lifetime measurements show high actuator reliability at medium actuation voltages, with 100 million cycles and a 10-day down-state test successfully completed.

\section{ACKNOWLEDGMENT}

The research described herein is a collaboration effort between KTH Royal Institute of Technology, Stockholm, Sweden, and the Jet Propulsion Laboratory, California Institute of Technology, Pasadena, California, USA, under contract with National Aeronautics and Space Administration.

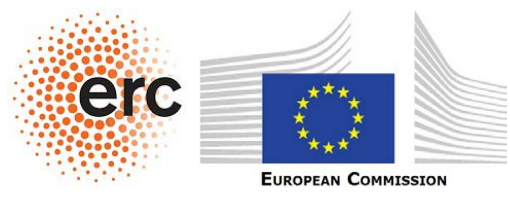

The contribution by KTH to this work has received funding from the European Research Council (ERC) under the European Union's Horizon 2020 research and innovation programme (grant agreement No 616846), The Swedish Foundation for Strategic Research Synergy Grant Electronics SE13-007, and through a Nils and Hans Backmark scholarship. 


\section{REFERENCES}

[1] C. A. Leal-Sevillano, T. J. Reck, C. Jung-Kubiak, G. Chattopadhyay, J. A. Ruiz-Cruz, J. R. Montejo-Garai, and J. M. Rebollar, "Silicon micromachined canonical E-plane and H-plane bandpass filters at the terahertz band," IEEE Microw. Wireless Compon. Lett., vol. 23, no. 6, pp. 288-290, Jun. 2013.

[2] W. R. McGrath, C. Walker, M. Yap, and Y.-C. Tai, "Silicon micromachined waveguides for millimeter-wave and submillimeter-wave frequencies," IEEE Microw. Guided Wave Lett., vol. 3, no. 3, pp. 6163, Mar. 1993.

[3] V. M. Lubecke, K. Mizuno, and G. M. Rebeiz, "Micromachining for terahertz applications," IEEE Trans. Microw. Theory Tech., vol. 46, no. 11, pp. 1821-1831, Nov. 1998.

[4] F. Boussaha, J. Kawamura, J. Stern, and C. Jung-Kubiak, " $2.7 \mathrm{THz}$ balanced waveguide HEB mixer," IEEE Trans. THz Sci. Technol., vol. 4, no. 5, pp. 545-551, Sep. 2014.

[5] "COM DEV International." [Online]. Available: http://www.meslmicrowave.com/Products_Catalogue/Rotary_Switches/ Waveguide_Rotary_Switches.aspx [Jul. 1, 2016].

[6] "Quinstar Technology Inc." [Online]. Available: http://quinstar.com/ millimeter-wave/waveguide-pin-switch-qssqsd-series [Jul. 1, 2016].

[7] G. M. Rebeiz, C. D. Patel, S. K. Han, C.-H. Ko, and K. M. J. Ho, "The search for a reliable MEMS switch," IEEE Microw. Mag., vol. 14, no. 1, pp. 57-67, 2013.

[8] H. Zareie and G. M. Rebeiz, "Compact high-power SPST and SP4T RF MEMS metal-contact switches," IEEE Trans. Microw. Theory Tech., vol. 62, no. 2, pp. 297-305, Feb. 2014.

[9] G. M. Rebeiz, RF MEMS Theory, Design, and Technology. NewYork: Wiley, 2003

[10] K. B. Cooper and G. Chattopadhyay, "Submillimeter-wave radar: solidstate system design and applications," IEEE Microw. Mag., vol. 15, no. 7, pp. 51-67, 2014.

[11] M. Daneshmand and R. R. Mansour, "RF MEMS satellite switch matrices," IEEE Microw. Mag., vol. 12, no. 5, pp. 92-109, Aug. 2011.

[12] F. Ke, J. Miao, and J. Oberhammer, "A ruthenium-based multimetalcontact RF MEMS switch with a corrugated diaphragm," J. Microelectromech. Syst., vol. 17, no. 6, pp. 1447-1459, Dec. 2008.

[13] L. Chen, Z. J. Guo, N. Joshi, H. Eid, G. G. Adams, and N. E. McGruer, "An improved SPM-based contact tester for the study of microcontacts," J. Micromech. Microeng., vol. 22, no. 4, Mar. 2012.

[14] M. Daneshmand, R. R. Mansour, and N. Sarkar, "RF MEMS waveguide switch," IEEE Trans. Microw. Theory Tech., vol. 52, no. 12, pp. 26512657, Dec. 2004.

[15] M. Daneshmand and R. R. Mansour, "Multiport MEMS-based waveguide and coaxial switches," IEEE Trans. Microw. Theory Tech., vol. 53, no. 11, pp. 3531-3537, Nov. 2005.

[16] N. Vahabisani and M. Daneshmand, "Monolithic millimeter-wave MEMS waveguide switch," IEEE Trans. Microw. Theory Tech., vol. 63, no. 2, pp. 340-351, Feb. 2015.

[17] Z. Baghchehsaraei, U. Shah, J. Åberg, G. Stemme, and J. Oberhammer, "MEMS reconfigurable millimeter-wave surface for V-band rectangularwaveguide switch," Int.J. Microw. Wireless Technol., vol. 5, no. 3, pp. 341-349, Jun. 2013.

[18] Z. Baghchehsaraei and J. Oberhammer, "Parameter analysis of millimeter-wave waveguide switch based on a MEMS reconfigurable surface," IEEE Trans. Microw. Theory Tech., vol. 61, no. 12, pp. 43964404, Dec. 2013.

[19] U. Shah, E. Decrossas, C. Jung-Kubiak, T. Reck, G. Chattopadhyay, I. Mehdi, and J. Oberhammer, "Submillimeter-wave 3.3-bit RF MEMS phase shifter integrated in micromachined waveguide," IEEE Trans. THz Sci. Technol., vol. 6, no. 5, pp. 33-38, Sep. 2016.

[20] U. Shah, T. Reck, E. Decrossas, C. Jung-Kubiak, H. Frid, G. Chattopadhyay, I. Mehdi, and J. Oberhammer, "500-750 GHz submillimeter-wave MEMS waveguide switch," in IEEE MTT-S Int. Microw. Symp. Dig., May 2016, pp. 1-4.

[21] T. Vähä-Heikkilä and M. Ylönen, "G-band distributed microelectromechanical components based on CMOS compatible fabrication," IEEE Trans. Microw. Theory Tech., vol. 56, no. 3, pp. 720-728, Mar. 2008.

[22] T. J. Reck, C. Jung-Kubiak, and G. Chattopadhyay, "A 700-GHz MEMS waveguide switch," IEEE Trans. THz Sci. Technol., vol. 6, no. 4, pp. 641-643, Jul. 2016

[23] T. J. Reck, C. Jung-Kubiak, J. Gill, and G. Chattopadhyay, "Measurement of silicon micromachined waveguide components at 500-750 GHz," IEEE Trans. THz Sci. Technol., vol. 4, no. 1, pp. 33-38, Jan. 2014.

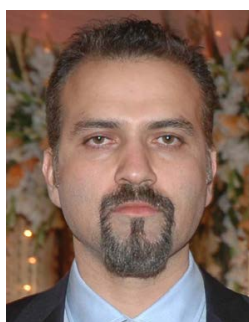

Umer Shah (S'09-M'14) was born in 1981 in Pakistan. He received his BS degree in Engineering from GIK Institute Pakistan in 2003, Master of Science degree in Wireless Engineering from the Technical University of Denmark (DTU) in 2007 and $\mathrm{PhD}$ degree in Microsystem Technology from KTH Royal Institute of Technology, Stockholm, Sweden in 2014. Since May 2014, he is a Postdoctoral researcher in the Micro and Nanosystems group at KTH Royal Institute of Technology. His research focuses includes RF MEMS based filters, phase shifters, matching circuits and antennas.

Umer has authored and co-authored more than 30 reviewed research papers. $\mathrm{He}$ is the recipient of the Best Student Paper Award presented at Asia Pacific Microwave Conference 2010, Yokohama, Japan. Umer is also the recipient of the "2014 IEEE MTT Graduate Fellowship Award" for his research activities.

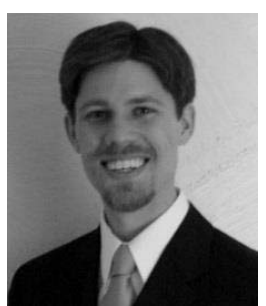

Theodore Reck (M'04-SM'15) received the B.S. degree in electrical engineering from the University of Texas at Austin in 2000 and PhD in Electrical Engineering at the University of Virginia in 2010. From 2010 to 2013 he was a NASA postdoctoral fellow at the Jet Propulsion Laboratory (JPL) designing terahertz devices that utilize silicon micromachining.

Presently he is a member of the technical staff at JPL. His research interests also include RF-MEMS, antenna arrays, terahertz metrology, and cryogenic MMIC LNAs.

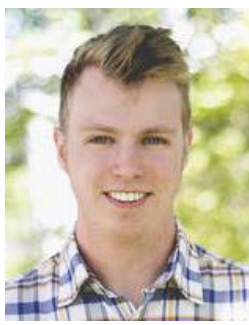

Henrik Frid (S'13) received the B.Sc. and M.Sc. degrees in Engineering Physics (with a specialization in electromagnetic theory, antenna theory, and electromagnetic wave propagation and scattering) from the KTH Royal Institute of Technology, Stockholm, Sweden, in 2015, where, since August 2014, he has been pursuing the Ph.D. degree in electrical engineering. Parallel to his studies, he worked as a Research Assistant at KTH on the topic of electrohydrodynamic flow simulations for liquid highvoltage insulators. During 2013, he joined Saab, in Linköping Sweden, for an internship on polarimetric radar calibration, and in 2014, he did a M.Sc. thesis project at Saab in Järfälla (Sweden), on calculating the mutual coupling between antennas on an aircraft. His current research interests include electromagnetic theory, reconfigurable antennas, and RF-THz MEMS-based switches and antennas. Mr. Frid received the 2012 Multiphysics Student Award and he was selected for the 2014 Excellence Doctoral Program at KTH.

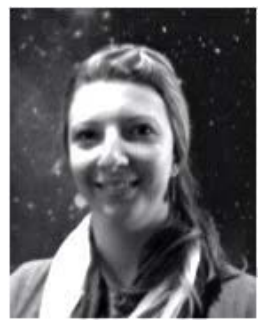

Cecile Jung-Kubiak (M'15) received a masters degree in Physics and Materials Chemistry from PolytechMontpellier, France in 2006 and the $\mathrm{PhD}$ degree in Physics from Universite Paris-Sud XI, France in 2009. She was the recipient of a 2-year NASA Postdoctoral Fellowship at the California Institute of Technology in 2010, and she is now a member of the Technical Staff for the S.W.A.T. group of the Jet Propulsion Laboratory, in Pasadena, USA. Her research interests include the development of silicon micromachining technologies using DRIE techniques, the miniaturization of multi-pixel arrays to build compact 3-D instruments and GaAs-based frequency multipliers and mixers in the $\mathrm{THz}$ region. She has coauthored over 50 papers in international journals and conferences, holds several patents and was a recipient of the 2010 JPL Outstanding Postdoctoral Research Award in the field Technology, Instrumentation, and Engineering and the 2014 IEEE THz Science and Technology Best Paper Award. 


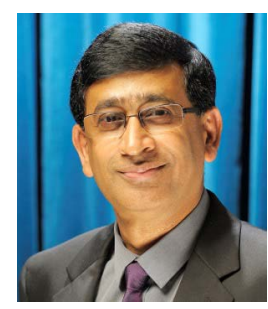

Goutam Chattopadhyay (S'93-M'99-SM'01-F'11) is a Senior Research Scientist at the NASAs Jet Propulsion Laboratory, California Institute of Technology, and a Visiting Associate at the Division of Physics, Mathematics, and Astronomy at the California Institute of Technology, Pasadena, USA. He received the B.E. degree in electronics and telecommunication engineering from the Bengal Engineering College, Calcutta University, Calcutta, India, in 1987, the M.S. degree in electrical engineering from the University of Virginia, Charlottesville, in 1994, and the Ph.D. degree in electrical engineering from the California Institute of Technology (Caltech), Pasadena, in 1999. From 1987 until 1992, he was a Design Engineer with the Tata Institute of Fundamental Research (TIFR), Pune, India.

His research interests include microwave, millimeter-, and submillimeterwave heterodyne and direct detector receivers, frequency sources and mixers in the terahertz region, antennas, SIS mixer technology, direct detector bolometer instruments; InP HEMT amplifiers, mixers, and multipliers; high frequency radars, and applications of nanotechnology at terahertz frequencies. He has more than 250 publications in international journals and conferences and holds more than fifteen patents. Among various awards and honors, he was the recipient of the Best Undergraduate Student Award from the University of Calcutta in 1987, the Jawaharlal Nehru Fellowship Award from the Government of India in 1992, and the IEEE MTT-S Graduate Fellowship Award in 1997. He was the recipient of the best journal paper award in 2013 by IEEE Transactions on Terahertz Science and Technology, and IETE Prof. S. N. Mitra Memorial Award in 2014. He also received more than 30 NASA technical achievement and new technology invention awards. He is an associate editor of the IEEE Transactions on Antennas and Propagation, a Fellow of IEEE (USA) and IETE (India) and an IEEE Distinguished Lecturer.

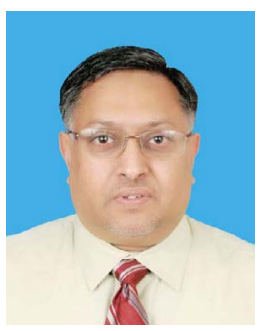

Imran Mehdi (S'83-M'90-SM'05-F'10) received the B.S.E.E., M.S.E.E., and Ph.D. degrees from the University of Michigan, MI, USA, in 1985, 1986, and 1990, respectively.

He is a Senior Research Scientist at the Jet Propulsion Laboratory, California Institute of Technology. He joined JPL in 1990 and is currently a Group Supervisor in the Instrument Electronics and Sensors Section. His responsibilities include developing $\mathrm{THz}$ components, technologies and subsystems for current and future NASA missions. These devices and components were implemented on the ozone monitoring Microwave Limb Sounder (MLS) instrument which is still operational as well as the (Microwave Instrument on Rosetta Orbiter) MIRO instrument which represents the first submillimeter-wave receiver operational in deep space. From 1999 he led the effort of developing broadband solid-state sources from 200 to 2500 $\mathrm{GHz}$ for the Heterodyne Instrument for Far Infrared (HIFI) on the Herschel Space Observatory, a cornerstone European Space Agency mission. HIFI has successfully completed its mission enabling ground breaking astrophysics observations. He is an IEEE Fellow and serves as a topical editor for the IEEE Transactions on THz Science and Technology. His current interests include millimeter and submillimeter-wave devices and technology, nanotechnology, high-frequency instrumentation, 3-D Submm-wave systems, and development of compact, low-power heterodyne receivers for planetary missions.

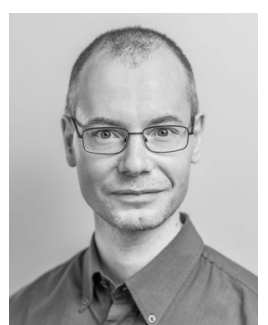

Joachim Oberhammer (M'06-SM'12), born in Italy in 1976; M.Sc. EE from Graz University of Technology, Austria, in 2000; Ph.D. from KTH Royal Institute of Technology in Stockholm, Sweden, in 2004. Post-doctoral research fellow at Nanyang Technological University, Singapore, in 2004, and at Kyoto University, Japan, in 2008. Since 2005 leading radio-frequency/microwave/terahertz micro-electromechanical systems research at $\mathrm{KTH}$; Associate Professor at KTH in 2010; Professor in Microwave and $\mathrm{THz}$ Microsystems at KTH since 2015. Guest researcher at Nanyang Technological University, Singapore, in 2007; guest researcher at NASA-Jet Propulsion Laboratory, USA, in 2014.

$\mathrm{He}$ is author and co-author of more than 100 reviewed research papers and holds 4 patents. In 2004, 2007, and 2008 he got an award by the Ericsson Research Foundation, a grant by the Swedish Innovation Bridge, and a scholarship by the Japanese Society for the Promotion of Science, respectively. The research work he is heading received six Best Paper Awards (five of which at IEEE conferences), and four IEEE Graduate Fellowship Awards (by MTT-S and by AP-S) since 2009. He served as TPRC member of IEEE Transducers 2009 and 2015, IEEE International Microwave Symposiums 2010-2016, IEEE Micro Electro Mechanical Systems 2011 and 2012, and IEEE Radio and Wireless Week 2015 and 2016. Dr Oberhammer is Steering Group member of the IEEE MTT-S and AP-S Chapers Sweden since 2009. In 2013, he received an ERC Consolidator Grant by the European Research Council. Since 2014 he is Steering Group Member of the Young Academy of Sweden. 